\title{
Open
}

\section{Maternal phenylketonuria syndrome: studies in mice suggest a potential approach to a continuing problem}

\author{
William L. Zeile ${ }^{1}$, Helen C. McCune ${ }^{2}$, Donald G. Musson ${ }^{3}$, Brian O'Donnell ${ }^{1}$, Charles A. O'Neill ${ }^{3}$, Laurie S. Tsuruda ${ }^{3}$, \\ Roberto T. Zori ${ }^{2}$ and Philip J. Laipis ${ }^{1}$
}

BACKGROUND: Untreated phenylketonuria (PKU), one of the most common human genetic disorders, usually results in mental retardation. Although a protein-restricted artificial diet can prevent retardation, dietary compliance in adults is often poor. In pregnant PKU women, noncompliance can result in maternal PKU syndrome, where high phenylalanine (Phe) levels cause severe fetal complications. Enzyme substitution therapy using Phe ammonia lyase (PAL) corrects PKU in BTBR Phe hydroxylase (Pah ${ }^{\text {enu2 }}$ ) mutant mice, suggesting a potential for maternal PKU syndrome treatment in humans.

METHODS: We reviewed clinical data to assess maternal PKU syndrome incidence in pregnant PKU women. We treated female PKU mice (on normal diet) with PAL, stabilizing Phe at physiological levels, and mated them to assess pregnancy outcomes.

RESULTS: Patient records show that, unfortunately, the efficacy of diet to prevent maternal PKU syndrome has not significantly improved since the problem was first noted 40 years ago. PAL treatment of pregnant PKU mice shows that offspring of PAL-treated dams survive to adulthood, in contrast to the complete lethality seen in untreated mice, or limited survival seen in mice on a PKU diet.

CONCLUSION: PAL treatment reduced maternal PKU syndrome severity in mice and may have potential for human PKU therapy.

D henylketonuria (PKU), perhaps the most common inborn error of metabolism, results from a partial or complete lack of phenylalanine (Phe) hydroxylase (PAH) enzymatic activity. The resulting inability to convert dietary Phe to tyrosine (Tyr) leads to varying degrees of hyperphenylalaninemia (1). Phe accumulation to high levels $(>1000 \mu \mathrm{M})$ in blood, as in "classic PKU" patients, is usually neurotoxic. If untreated, PKU patients can exhibit a variety of serious clinical effects, including diminished cognitive function, growth failure, microcephaly, and developmental delay. A significant inverse correlation between blood Phe level and intelligence in patients with PKU has been established (2). Bickel et al.'s (3) concept of dietary therapy led to prevention of many of the deleterious effects of hyperphenylalaninemia. However, successful early diagnosis by newborn screening and PKU dietary treatment has not fully addressed the serious problem of maternal PKU syndrome (4). Female PKU patients, who may have adhered to dietary treatment during childhood, often relax their compliance during adulthood. Their elevated plasma Phe concentrations can become an acute problem during pregnancy.

The developing fetus in mothers with poorly controlled PKU can be affected by intrauterine exposure to elevated Phe levels. The Maternal Phenylketonuria Collaborative Study established that elevated maternal blood Phe levels are teratogenic (5-9). Children born with maternal PKU syndrome suffer from microcephaly, craniofacial dysmorphism, low birth weight, congenital heart disease, developmental delays, and mental retardation (10-12). In 1980, among 524 pregnancies in 155 women, $95 \%$ of mothers with blood Phe concentrations $\geq 1,200 \mu \mathrm{M}$ had at least one mentally retarded child (13). The frequency of mental retardation, microcephaly, and congenital heart disease occurred at far greater frequencies than in the general population, and correlated with maternal blood Phe concentrations.

Fetal outcomes in pregnant women with PKU were substantially improved by strict adherence to Phe-restricted diets (14). In one report, women who achieved blood Phe concentrations of $120-360 \mu \mathrm{M}$ by week 8 of gestation had normal outcomes (15). Optimum outcomes were achieved when women initiated a Phe-restricted diet before conception (16-18); however, in cases of unplanned pregnancy, the mother may be unaware of her pregnancy for 4-5 weeks and thus there is no change in dietary Phe intake (12). In addition, the challenges of maintaining a strict PKU diet before and during pregnancy, poor access to medical care, financial constraints, and psychosocial issues $(11,19)$ make dietary restriction a difficult path for many PKU women. Our current experience in managing female PKU patients at risk for maternal PKU syndrome, summarized below, shows that nearly $90 \%$ of "at-risk" pregnancies fail at achieving good Phe control on diet alone. This led us to investigate the potential of enzyme substitution therapy, using a recombinant form of

\footnotetext{
${ }^{1}$ Department of Biochemistry and Molecular Biology, University of Florida College of Medicine, University of Florida Academic Health Center, Gainesville, Florida; ${ }^{2}$ Department of Pediatrics, University of Florida College of Medicine, Gainesville, Florida; ${ }^{3}$ BioMarin Pharmaceutical Inc., Novato, California. Correspondence: Philip J. Laipis (plaipis@ufl.edu) Received 19 April 2017; accepted 30 November 2017; advance online publication 31 January 2018. doi:10.1038/pr.2017.323
} 


\section{Articles | zeile et al.}

Phe ammonia lyase (pegvaliase, formerly rAvPAL-PEG), (20) for treating maternal PKU syndrome in the BTBR Pah ${ }^{\text {enu2 }}$ (PKU) mouse (21).

The PKU mouse is an established model of human PKU and exhibits clinical characteristics similar to PKU patients, including hyperphenylalanemia (baseline plasma Phe concentrations of 1,000 to $2,000 \mu \mathrm{M})$, hypopigmentation, cognitive defects, audiogenic seizures, and maternal PKU syndrome $(21,22)$. In previous studies, a single, weekly subcutaneous administration of pegvaliase at doses of $80 \mathrm{mg} / \mathrm{kg}$ to male PKU mice lowered plasma Phe levels from $\sim 1,200 \mu \mathrm{M}$ to $<200 \mu \mathrm{M}$ for up to 10 months (23). Results presented here show pegvaliase treatment of pregnant female PKU mice resulted in greatly improved fetal outcomes, suggesting that such an approach may be useful in the treatment of human maternal PKU syndrome.

\section{METHODS}

Complete methods are in the Supplementary Data.

\section{Retrospective Clinical Review of Records}

This study was approved by the University of Florida Institutional Review Board, Project Number 131-2011. Records of 28 pregnancies were initially reviewed and summarized by HCM; clinical data were then verified by either P.J.L. or R.T.Z. Recorded values for blood Phe levels came from dried blood spots, measured at the time of collection by various clinical laboratories.

\section{Animals, Care, and Procedures}

Wild-type (WT) animals of the BTBR inbred strain, heterozygous BTBR Pah ${ }^{\text {enu2 }}$ carrier mice (HET), and homozygous BTBR Pah ${ }^{\text {enu2 }}$ mutant (PKU) mice (21) were originally from Jackson Laboratories (Bar Harbor, ME). All protocols were approved by the University of Florida IACUC. For pegvaliase treatment, mice were restrained and pegvaliase was injected subcutaneously into the upper back-lower neck area. Plasma Phe was determined from a $45 \mu$ l blood sample, collected into a heparinized microcapilliary tube.

\section{PEGylated Recombinant Phe Ammonia Lyase}

A PEGylated, recombinant form of Anabaena variabilis pegvaliase was provided by BioMarin Pharmaceutical (Novato, CA). A sterile solution of $10 \mathrm{mg} / \mathrm{ml}(\sim 12 \mathrm{IU} / \mathrm{ml})$ pegvaliase was diluted to $5 \mathrm{mg} / \mathrm{ml}$ weekly and stored at $0{ }^{\circ} \mathrm{C}$. Aliquots were diluted to $0.5-2.0 \mathrm{mg} / \mathrm{ml}$, depending on desired dose, and $10 \mu \mathrm{l} / \mathrm{g}$ body weight injected.

\section{Phe Assay}

Plasma Phe was determined using a fluorimetric assay (24), extensively modified for small volumes, rapid turnaround, and microtiter plate format. Plasma from blood samples was frozen until assay. An aliquot $(7.5 \mu \mathrm{l})$ of each plasma sample was deproteinized, placed in a 96-well PCR plate with assay cocktail, and incubated at $60{ }^{\circ} \mathrm{C}$ for $2 \mathrm{~h}$. Copper reagent was added to each well of a 96-well $0.5 \mathrm{ml}$ black fluorimeter plate (Nunc, Roskilde, Denmark, critical for accurate plasma Phe determination), and the incubated plasma samples were added. The plate was read on a SpectraMax M2 platereader (Molecular Devices, Sunnyvale, CA), with parameters of excitation: $378 \mathrm{~nm}$; emission: $512 \mathrm{~nm}$.

\section{Amino-Acid Analysis}

Individual amino acids and standards (Sigma), and high-performance liquid chromatography reagents (Fisher), were obtained from Sigma Chemical (St Louis, MO) and Fisher Scientific (Atlanta, GA). Plasma samples were deproteinized and norleucine was added as an internal standard. The dried samples were derivatized with phenylthiocarbamyl, vacuum dried, excess reagent removed, and chromatographed on a Beckman System Gold LC with UV-VIS detector $(254 \mathrm{~nm})$ and a Phenomenex reversed-phase C18 analytical column. Amino-acid concentrations were calculated by using standard/internal standard peak areas compared with sample peak areas.

\section{Pharmacodynamic Modeling}

Pharmacokinetic parameters were determined from single-dose pharmacokinetic/pharmacodynamic studies (Musson and Laipis, data not shown) in naïve mice. Plasma samples were analyzed for pegvaliase concentrations by BioMarin Pharmaceutical using a pegvaliase-specific sandwich enzyme-linked immunosorbent assay. Bound pegvaliase was detected with an anti-PEG antibody. Limit of quantitation was $60 \mathrm{ng} / \mathrm{ml}$. Plasma Phe levels were determined as described above. Plasma Phe plasma concentrations at different pegvaliase dose regimens were modeled using WinNonlin software version 5.2 (Certara, Princeton, NJ, USA).

\section{RESULTS}

\section{Maternal PKU: The University of Florida College of Medicine Experience}

The University of Florida College of Medicine, Department of Pediatrics, Division of Clinical Genetics and Metabolism, serves the north Florida region from Pensacola to Jacksonville to Orlando. It is a university medical center where most PKU patients in the area receive treatment for their disorder. Pregnant PKU women receive prenatal counseling and intensive nutritional therapy because of the risks to the fetus. Although distance from the center makes regular visits impractical for many patients, they receive ongoing nutritional counseling and are encouraged to submit weekly blood Phe levels and body weights. Our recommendations to pregnant (and prospective pregnant) PKU women are to maintain blood Phe levels between 120 and $240 \mu \mathrm{M}$ and Tyr above $50 \mu \mathrm{M}$, to meet the recommended nutrient intake for pregnancy and to promote adequate weight gain.

We retrospectively examined records of 28 pregnancies, occurring between 1996 and 2010. Patients were classified into three groups:

Group 1:Excellent Phe Control (3/28) - average blood Phe levels were below $360 \mu \mathrm{M}$ throughout gestation, with maximum values below $420 \mu \mathrm{M}$ Phe at any time and at least six recorded Phe values in the first trimester.

Group 2: Marginal Phe Control (11/28)—average blood Phe levels were below $420 \mu \mathrm{M}$ during the first trimester then subsequently averaged below $360 \mu \mathrm{M}$ during the second and third trimesters. Patients presented with an initial plasma Phe value of $700 \mu \mathrm{M}$ or less in the first trimester and had two or fewer values above $500 \mu \mathrm{M}$ during the remainder of the pregnancy.

Group 3: Inadequate Phe Control (14/28) - average plasma Phe levels were above $420 \mu \mathrm{M}$ in the first trimester, with average values above $360 \mu \mathrm{MM}$ during the remainder of gestation and/or with multiple values above $420 \mu \mathrm{M}$.

Average Phe levels over the 40 weeks of pregnancy for the three patient cohorts are shown in Supplementary Figure S1 online. The outcome of each pregnancy, classified as normal or affected, based on birth parameters (weight, height, head circumference), available clinical exam information, and 
presence of conditions associated with maternal PKU syndrome, are summarized in Supplementary Table S1 There are distinct difference between the groups (absolute Phe level, degree of control achieved, maximum Phe levels recorded during each time interval, and pregnancy outcomes); a detailed description of each group is in the file "Supplementary Clinical Outcome".

The difficulties in following proper diet were clearly documented in these patient groups. Only 11\% (3/28) of all PKU women were in good Phe control before pregnancy. Despite intensive counseling about recommended blood Phe levels and submitting blood Phe levels with diet histories, fewer than half of the women submitted blood samples before the sixth week of gestation or provided sufficient weekly blood samples to properly monitor Phe levels. Less than half of the blood Phe levels received were within 120-240 $\mu \mathrm{M}$. Twentyfive percent of women $(7 / 28)$ did not return to clinic after their pregnancy nor did they provide birth parameters of the infant. Birth outcomes are also suboptimal; of 28 pregnancies, only 12 had normal weight, height, and head circumference at birth. A total of 11 infants were affected, including 9 with microcephaly (Supplementary Table S1), and in 5 cases there was no information about the infant's outcome. We also lack 1 year and subsequent developmental data on most pregnancies, and it is possible that some normal infants had a less than optimum developmental outcome. These problems are exacerbated in the most poorly compliant Group 3 patients. Lack of follow-up is a continuing problem in the management of patients at risk for maternal PKU syndrome. Our results are consistent with previous published reports, and demonstrate that the current recommended dietary management practice does not function well in a significant fraction of the PKU population during pregnancy.

\section{Pegvaliase and the PKU Mouse}

To assess the potential therapeutic benefit of pegvaliase in treating both pregnant PKU women and their fetuses at risk for maternal PKU, we examined its effects on maternal PKU syndrome in female PKU mice, using varied doses and treatment schedules. The results of our studies are summarized in Table 1; detailed results for each study are shown in Supplementary Table S2. We note here that maternal PKU syndrome in the PKU mouse is significantly more severe than in human PKU patients, complete neonatal death within the first $24 \mathrm{~h}$ of life is the usual outcome (21). In addition, although a Phe-restricted diet can partially correct maternal PKU syndrome in PKU mice, few pups survive to weaning on a low Phe diet (21).

Initial studies in male PKU mice demonstrated that weekly subcutaneous administration of $80 \mathrm{mg} / \mathrm{kg}$ pegvaliase resulted

Table 1. Dose range, plasma Phe, and pregnancy in pegvaliase-treated PKU female mice

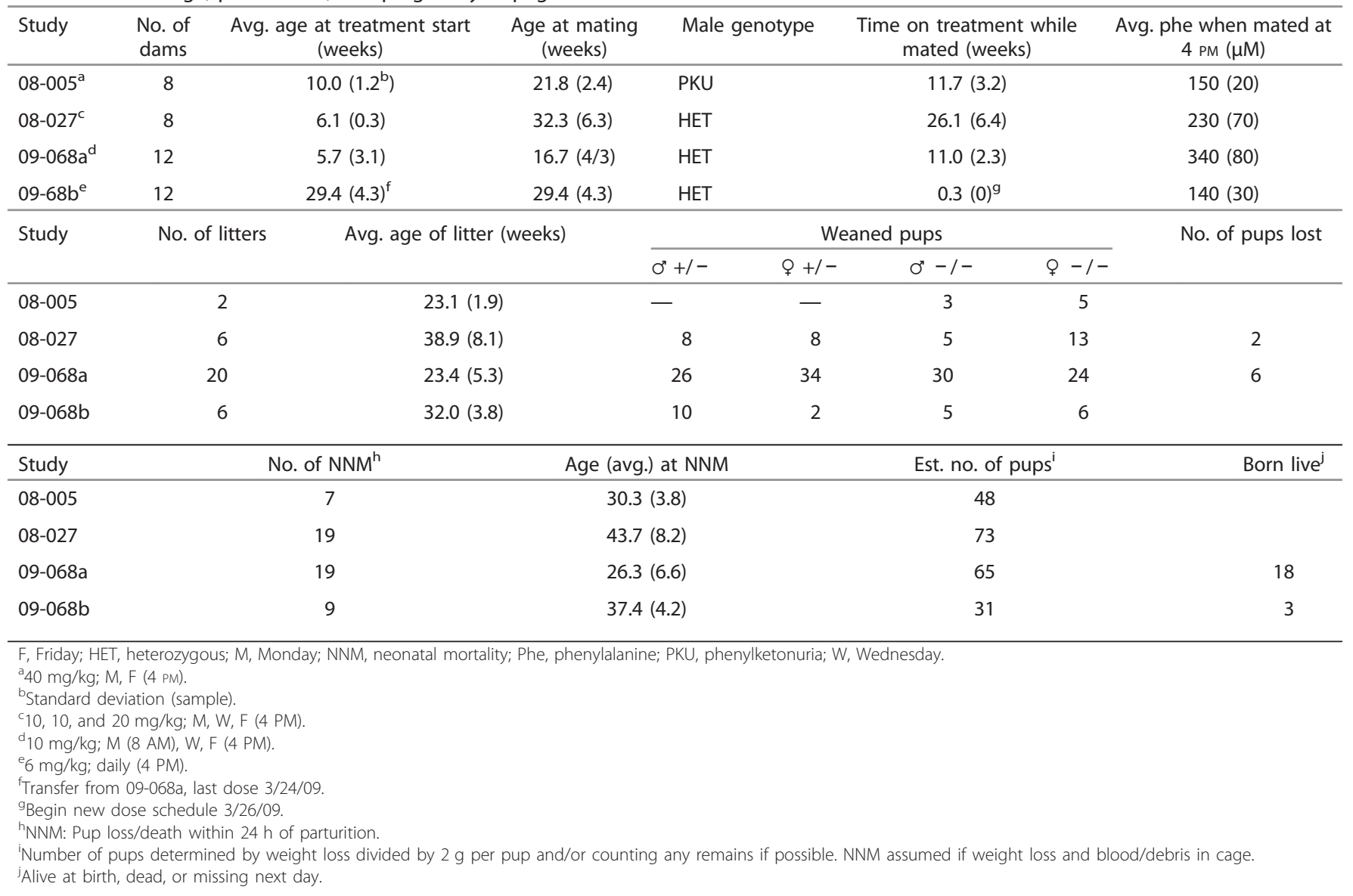




\section{Articles | zeile et al.}

in $4 \mathrm{PM}$ (baseline) plasma Phe levels of approximately physiological levels $(50-150 \mu \mathrm{M})$ for the duration of the week, once the initial attenuated response, most likely due to an antibody response, had abated (23). This $80 \mathrm{mg} / \mathrm{kg}$ weekly dose schedule was effective for only 4-5 days in female PKU mice, with 4 PM Phe levels rising to $1,000 \mu \mathrm{M}$ or above by the end of a week, likely due to the higher gender-specific plasma Phe levels of females compared with males (Laipis et al., data not shown). A total weekly dose of $80 \mathrm{mg} / \mathrm{kg}$, split into two $40 \mathrm{mg} / \mathrm{kg}$ per doses administered twice per week, Monday and Friday, reduced 4 PM (predose) Phe levels to near physiological over the full week. Female PKU mice (Study 08-005), on this protocol, could become pregnant and gave birth to live pups, although only two of nine pregnancies successfully weaned pups and both litters were small, 3 and 5 pups each (Table 1 and Supplementary Table S2). Other pregnancies resulted in neonatal mortality within $24 \mathrm{~h}$. The Phe levels of one PKU female mouse from Study 08-005 are in Supplementary Figure S2.

Multiple factors could contribute to the suboptimal response of murine maternal PKU syndrome to a pegvaliase treatment regimen that resulted in excellent growth, physical condition, and greatly extended lifespan in adult male and non-pregnant female PKU mice. First, single dose pharmacokinetic studies show that the half-life of pegvaliase in the PKU mouse model is $\sim 24 \mathrm{~h}$ (Sarkissian et al. (23); Laipis and Musson, unpublished). Two weekly doses, 72 and $96 \mathrm{~h}$ apart (the protocol in Study 08-005), could result in abnormally low plasma Phe immediately after a dose, which might not affect adults but could be deleterious to a developing fetus. Second, baseline plasma Phe levels are measured at $4 \mathrm{PM}$, a time when the nocturnal mice are generally inactive $(2 \mathrm{~h}$ before the end of the 6 Am to 6 PM light cycle). Mice awaken at 6 PM and begin an activity and feeding cycle that persists until the end of the dark cycle at $6 \mathrm{Am}$, this diurnal variation is examined below. Third, pregnancy weight gains are enormous, a $30 \mathrm{~g}$ female will weigh $\sim 50 \mathrm{~g}$ at day 20 of gestation and food intake will be more than double. Food intake drops back to normal after birth, but again rises during lactation as the nursing mother feeds what may be $40-50 \mathrm{~g}$ of pups at 14 days postpartum, when pups begin to sample solid food. These factors suggested the twice weekly dose schedules used in adult non-pregnant mice were not adequately maintaining steady physiological plasma Phe levels during pregnancy.

Initially, we suspected that maternal plasma Phe levels in Study 08-005 may have reached both too low and high extremes for pup survival, as the Phe-restricted diet study and the short pegvaliase half-life might imply (21,23). Accordingly, eight PKU female mice (Study 08-027) were sequentially treated with pegvaliase protocols designed by pharmacodynamics modeling to result in average plasma Phe levels of $\sim 250 \mu \mathrm{M}$ at $4 \mathrm{PM}$ (Table 1 and Supplementary Table S2). Although the final protocol (Monday, Wednesday, $10 \mathrm{mg} / \mathrm{kg}$; Friday, $20 \mathrm{mg} / \mathrm{kg}$ ) resulted in excellent physical condition and extended lifespan, only a modest increase in litter size and live pups (average litter size 6 (2 litters of 10 pups at birth); $32 \%$ of pregnancies weaned pups) was seen. These females were considerably older (Table 1 ) at the time of mating, due to protocol adjustments needed to reach the desired 4 PM plasma Phe level.

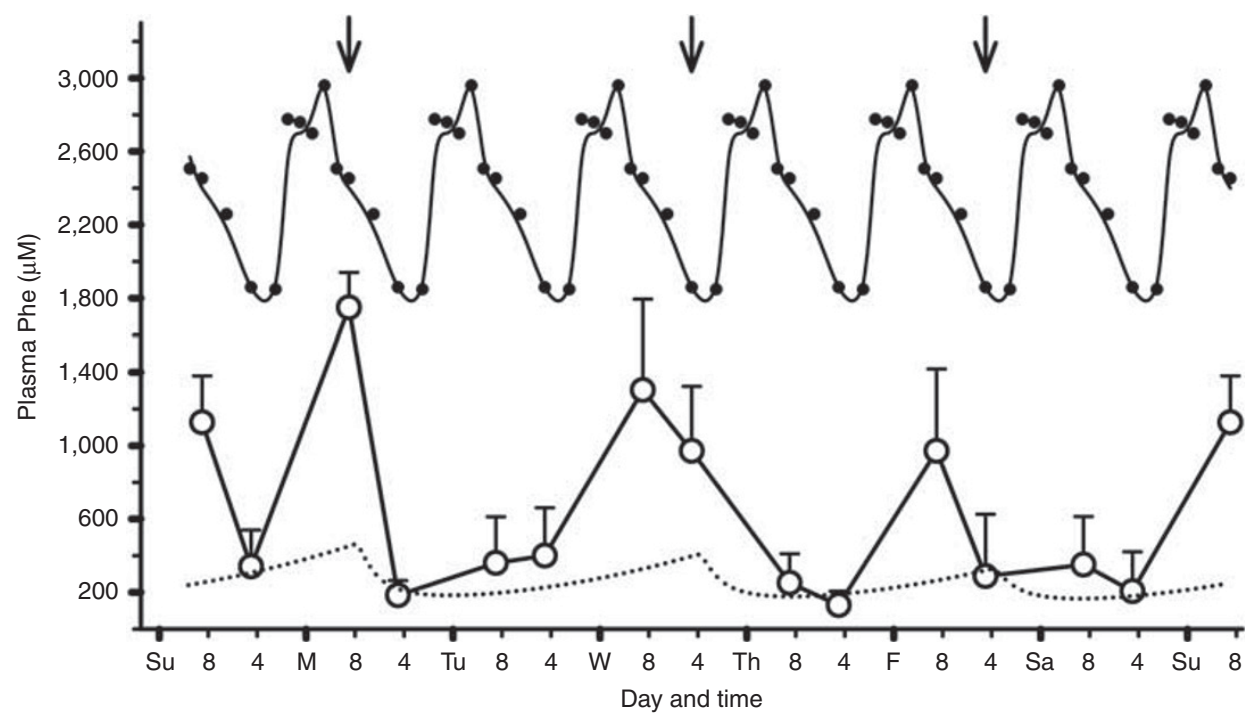

Figure 1. Diurnal variation in plasma phenylalanine (Phe) levels of pegvaliase-treated phenylketonuria (PKU) mice. Twelve female PKU mice (Study 09-068a) were injected subcutaneously three times weekly with $10 \mathrm{mg} / \mathrm{kg}$ pegvaliase (Monday, 8 AM; Wednesday, 4 PM; Friday, 4 PM; $\downarrow$ ). Pharmocodynamic modeling of this dose schedule, $(\cdots)$ predicted good regulation of plasma Phe levels. Blood samples $(40 \mu \mathrm{ll})$ were collected at 8 AM and 4 PM on different days of the week over a 7-week period and plasma Phe levels averaged to yield a composite weekly plasma Phe levels plot (०). The $x$-axis major hash marks represent days of the week (12 AM) and the minor hash marks indicate 8 AM and 4 PM. Composite data from untreated PKU female mice (Figure $\mathbf{2}, \bullet$ ) were repeated seven times to show peak correspondence with the weekly plasma Phe data from pegvaliase treatment. Plasma Phe peaks occur in the treated animals at times corresponding to maximum plasma Phe levels in untreated PKU mice, although marked reduction of 8 AM plasma Phe values are seen on days after a pegvaliase dose was administered. 
In a third study (09-068a), 12 PKU female mice were treated with a slightly modified dose schedule $(10 \mathrm{mg} / \mathrm{kg}$ pegvaliase, Monday 8 AM; Wednesday, Friday 4 PM), which pharmacodynamic modeling suggested should also provide uniform plasma Phe control (Figure 1 and Table 1). In addition, plasma samples were collected at $8 \mathrm{AM}$, in place of 4 PM samples. These12 PKU mice had an average of $300 \mu \mathrm{M}$ Phe levels at $4 \mathrm{PM}$, indicating good control of Phe levels during the week, as predicted by pharmacodynamic modeling. However, the levels in $8 \mathrm{Am}$ blood samples, were dramatically increased (Figure 1). These females were mated during this dose protocol and had a total of 39 pregnancies (Table 1, Supplementary Table S2). Slightly over 50\% of these pregnancies weaned pups, with an average litter size of about six pups (five litters of eight or more pups), which is better than previous results.

Clearly, pharmacodynamic modeling was not accurately representing physiology, thus while 09-68a was in progress, we examined plasma Phe levels in a group of six control PKU female mice, bled (over a period of 9 weeks) at 2 to $4 \mathrm{~h}$ intervals, to determine the diurnal fluctuation of plasma Phe levels as a function of food intake during the light-dark cycle (Figure 2). Plasma Phe levels increase by over $1000 \mu \mathrm{M}$ during the dark period, Phe levels then fall gradually during the daytime rest period, reaching a nadir between 4 and 8 PM. In contrast, two control groups (16 each) WT and HET female BTBR mice, bled at $8 \mathrm{AM}$ and $6 \mathrm{PM}$, had plasma Phe levels of $240 \pm 30 / 230 \pm 40 \mu \mathrm{M}$ and $280 \pm 30 / 210 \pm 40 \mu \mathrm{M}$, respectively, compared with the corresponding levels of 2,450 and 1,850 $\mu \mathrm{M}$ seen in PKU mice (Figure 2). A 30-g PKU mouse, fed ad libitum on standard 1\% Phe mouse chow,

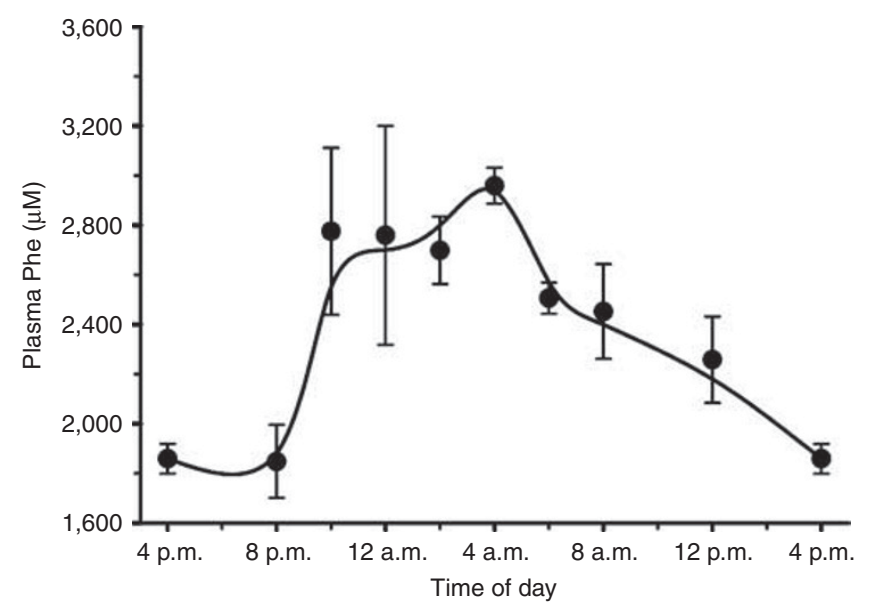

Figure 2. Diurnal variation in plasma phenylalanine (Phe) levels of untreated female phenylketonuria (PKU) mice. The change in plasma Phe levels that occurred during the $24 \mathrm{~h}$ dark-light cycle was determined from analysis of composite data. Blood samples $(40 \mu \mathrm{l})$ were obtained from six adult female PKU mice at the indicated times, with samples collected over a 9-week period to prevent stress from blood loss. Two samples were obtained from each animal at every time point. Plasma Phe results were combined and averaged to yield the composite $24 \mathrm{~h}$ data plot. The plot shows the fast rise of over $1,000 \mu \mathrm{M}$ in plasma Phe that occurred when the mice woke at 6 PM, began feeding, and the slower fall beginning at the 6 Am start of the light cycle. eats $\sim 25 \%$ of body weight per day, this results in a Phe intake of about $75 \mathrm{mg}$, many times sufficient to double plasma Phe levels. Superposition of the observed physiological plasma Phe levels in untreated PKU females (Figure 2) over the $8 \mathrm{AM}$ Phe levels of pegvaliase-treated female PKU mice in Figure 1 shows excellent correlation with the diurnal highs, except for the 8 AM time points $12-24 \mathrm{~h}$ post injection, when pegvaliase activity should still be high. As the $24 \mathrm{~h}$ half-life reduces active enzyme levels, pegvaliase enzyme activity that is sufficient to degrade excess Phe to physiologic levels during the daytime fast is unable to control the significantly higher levels present during the nighttime feeding period.

To eliminate this diurnal variation, the same 12 female PKU mice described above (Figure 1, 09-068a) were switched to a daily $6 \mathrm{mg} / \mathrm{kg}$ dose of pegvaliase (based on pharmacokinetic
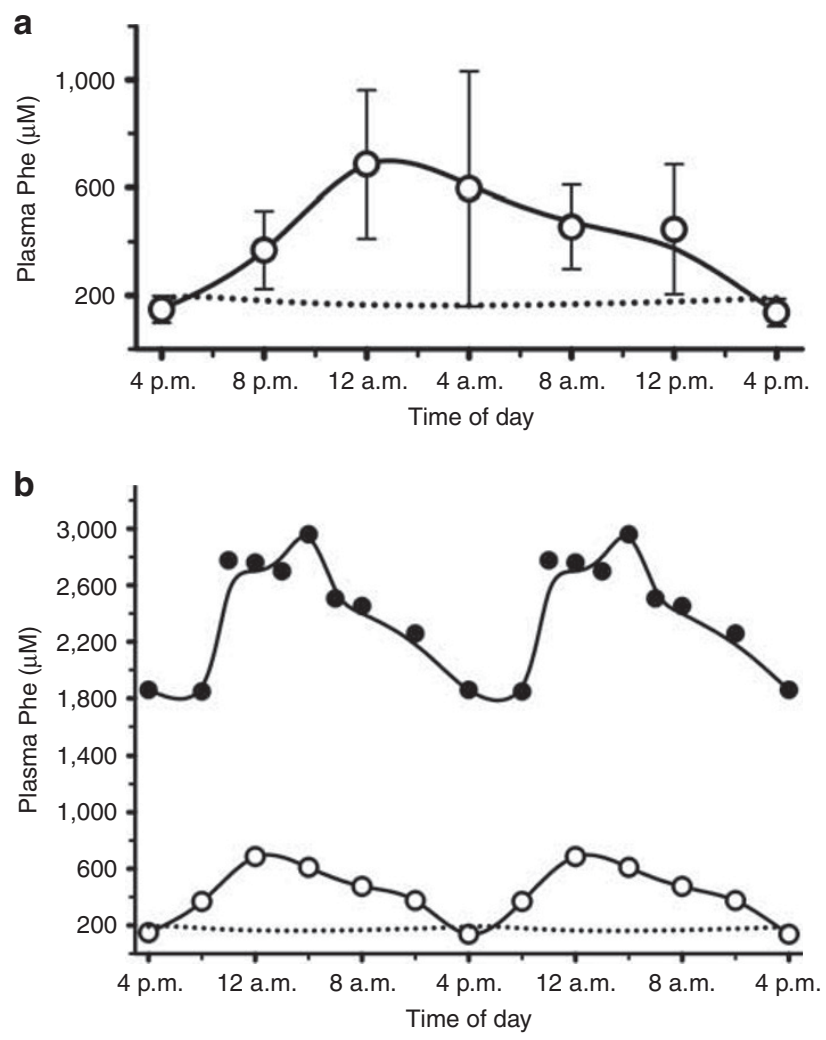

Figure 3. Diurnal variation in plasma phenylalanine (Phe) levels of pegvaliase-treated phenylketonuria (PKU) mice. (a) Female PKU mice (Study 09-068b) received daily subcutaneous injections of $6 \mathrm{mg} / \mathrm{kg}$ pegvaliase at 4 PM. In addition to the normal 4 PM baseline samples, additional blood samples were drawn at $4 \mathrm{~h}$ intervals over a 10 -week period to determine Phe levels throughout the light-dark cycle. Two plasma Phe values per animal per time point were averaged to create the $24 \mathrm{~h}$ composite profile $(0)$ shown in the figure. Pharmacodynamic modeling $(\cdots)$ gives an excellent match to the baseline 4 PM Phe values, but dietary intake results in a significant rise in plasma Phe that modeling does not simulate. (b) Two $24 \mathrm{~h}$ cycles of the composite data for untreated PKU female mice $(\bullet)$, from Figure 2, superimposed on two $24 \mathrm{~h}$ cycles of the $6 \mathrm{mg} / \mathrm{kg}$ daily dose ( $($ ) composite data from (a). There is excellent correspondence between dietary intake in untreated PKU mice and the pegvaliase-treated PKU mice, with marked, if incomplete, damping of the $1 \mathrm{mM}$ nightly increase seen in untreated animals. 


\section{Articles | zeile et al.}

parameters), to better match enzyme activity to dietary Phe levels (Figure 3a, 09-068b). As before, pharmacodynamic modeling again matched the baseline Phe levels. Importantly, although a diurnal increase in plasma Phe levels, which mirrors the condition in untreated PKU mice, (Figure $3 \mathbf{b}$ ) is still present, the peak magnitude is markedly reduced. There were 15 additional pregnancies during the 10 -week daily dosing protocol, of which six were successful (40\%, average litter size 4). Although these statistics are not equal to those of these females on the $3 \times /$ week dose protocol shown in Figure 1, their ages were now between 6 and 9 months at mating and 8.5 and 11 months at study end, very near the end of the reproductive lifespan (Supplementary Table S2). Interestingly, during the entire study, the six youngest females (pegvaliase treatment begun at 5 weeks of age or less) recorded 19 successful pregnancies, and 11 neonatal mortality events compared with the six older females (pegvaliase treatment begun at 6-12 weeks of age; 7 pregnancies; 15 neonatal mortality events).

One additional factor might affect maternal PKU syndrome treatment using pegvaliase. In untreated human PKU, the Tyr to Phe ratio is often decreased, with observed plasma levels of Tyr reduced to $50 \%$ of that seen in normal controls (25), this reduction might also affect maternal PKU outcomes. We measured Trp, Tyr, and Phe levels in WT, PKU, and pegvaliase-treated PKU mice by high-performance liquid chromatography (Figure 4). As in human PKU patients, plasma Tyr levels in the PKU mouse were reduced from normal levels, with no change in Trp levels. Reducing plasma

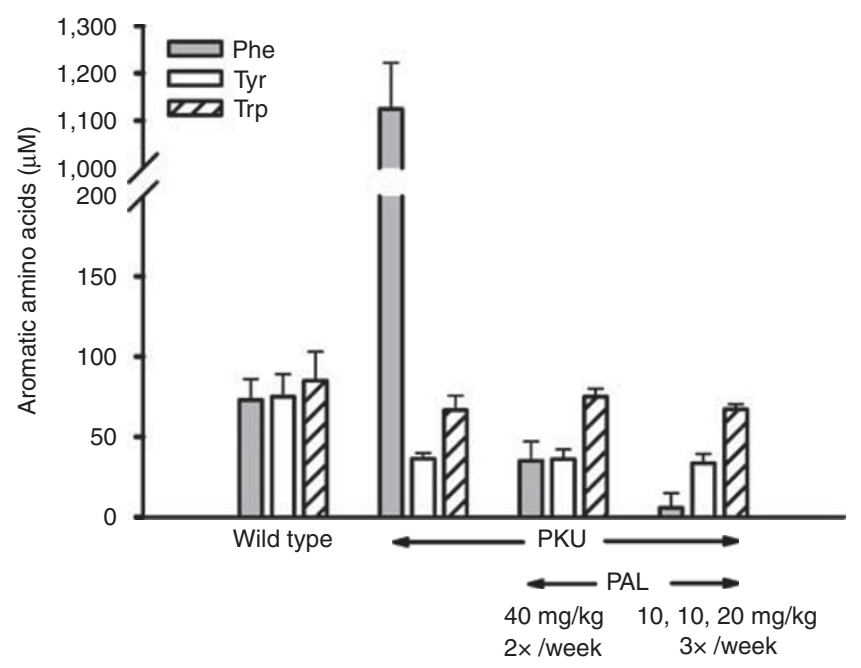

Figure 4. Aromatic amino-acid levels in plasma of wild-type (WT) and phenylketonuria (PKU) female mice. Plasma samples, obtained from untreated WT and PKU mice and pegvaliase-treated PKU mice, were analyzed by high-performance liquid chromatography (HPLC) separation. Plasma samples from untreated PKU mice show markedly increased levels in plasma phenylalanine (Phe) relative to WT mice, along with a $40-50 \%$ decrease in plasma tyrosine. Plasma tryptophan levels remained similar between the two genotypes. Pegvaliase treatment decreased plasma Phe to physiological or below normal physiological levels according to dose regimen. Tyrosine and tryptophan levels in untreated and pegvaliase-treated PKU mice were similar.
Phe levels from PKU to physiological or subphysiological levels by pegvaliase had no effect on plasma Tyr. Attempts to increase Tyr levels in the pegvaliase-treated PKU mouse by addition of Tyr to drinking water were unsuccessful because of its low solubility, hence, use of a Tyr-supplemented artificial diet (where low solubility will not limit potential uptake) will be necessary to determine the possible role of low Tyr in the partial response of maternal PKU syndrome in the PKU mouse.

\section{DISCUSSION}

Maternal PKU syndrome remains a problem of critical clinical importance, even though the cause is known and a treatment exists that prevents poor fetal outcomes. From 1996, even with the resources of a university medical center and a clinical geneticist-led team, 50\% of pregnant PKU women over a 15-year period could not control blood Phe levels during gestation, with most pregnancies in these women resulting in an affected child. None of the infants in this subset of patients were conceived with the mother at physiological Phe levels of 50-120 $\mu \mathrm{M}$. In addition to the poor compliance of these women with medical recommendations during their pregnancy, long-term follow-up on their infants was also difficult. The reasons for this failure of dietary treatment must include both the difficulty of adhering to a protein-restrictive diet and artificial amino-acid supplementation while pregnant, and a mother whose own cognitive ability may be impaired by her noncompliant diet. Other factors, such as a lack of social support, economic challenges, and a need for dietary supervision also affect the outcomes. Clearly, the unpleasant nature of the artificial diet is a major factor, and additional therapies for PKU that do not depend on diet are needed.

Maternal PKU syndrome in PKU mice is much more severe than in humans, with essentially $100 \%$ lethality by $24 \mathrm{~h}$ postpartum (21). This is in contrast to the birth of live, although affected infants, in uncontrolled PKU human mothers. Using a daily administration protocol of pegvaliase that considered steady-state Phe levels and the dietary Phe cycles shown in Figure 2, we were able to rescue $40 \%$ of pregnancies, despite a maternal age near the end of the reproductive lifespan. These results are very encouraging, as the normal outcome would be complete pup death. During the course of this protocol (13 weeks of $3 \times /$ weekly dosing and 15 weeks of daily dosing), two PKU mice had five successful litters each, equaling that of a wild-type female, and three of those litters were 10-11 pups, which is normal in the BTBR background strain. Although there were 26 successful pregnancies, there were also 26 neonatal mortality events. The detailed results (Supplementary Table S2) also suggest that the age at which the PKU female begins pegvaliase treatment is of critical importance for later reproductive success. About $63 \%$ of pregnancies in female PKU mice, which began their pegvaliase treatment between 2 and 5 weeks were successful, compared with $32 \%$ for females beginning treatment between 6 and 12 weeks of age. Hyperphenylalaninemia may be affecting 
proper development of both maternal behavior in young females and neonatal behavior in their pups via altered neurotransmitter metabolism, as discussed below.

Finally, these studies begin to address the role of dietary Phe in the maintenance of plasma Tyr levels in the mouse, and by extension, human patients. Tyr levels in PKU mice are approximately half of that seen in wild-type or heterozygous mice; similar effects of PKU on human Tyr levels have been reported $(25,26)$. This reduction in Tyr levels has been suggested to be caused by elevated plasma Phe preventing uptake and transport of dietary Tyr from the gut and across the intestinal epithelial cell to the plasma. We suggest instead that humans and mice have evolved to use the Tyr produced from Phe by PAH. Isotopic tracer studies have previously reported that a significant fraction of plasma Tyr is derived from Phe by action of PAH (27). Pegvaliase treatment of PKU mice offered an ideal means of examining this transport hypothesis, as it allowed us to reduce plasma Phe levels in the absence of PAH activity; the results of pegvaliase treatment provide a direct confirmation of the role of plasma Phe as a source of plasma Tyr. We also speculate that some of the maternal PKU syndrome severity in the PKU mouse might be due to chronic low Tyr levels affecting dopamine levels, which influence maternal behavior, nursing behavior, and appetite in the neonatal mouse (28-30). However, Trp levels in the brain have also been reported to be abnormally low in PKU patients and PKU mice (31), and an inhibitory effect of high plasma Phe levels on tryptophan hydroxylase has also been reported (32).

In conclusion, it is important to emphasize that maternal PKU syndrome in the PKU mouse is a significant challenge for treatment. The large diurnal plasma Phe level changes, significant weight gain during pregnancy, and the dietary intake needed for a lactating female to nurse her body weight and more in offspring make maintenance of a stable physiological Phe level extremely difficult. Indeed, use of a Phe-restricted diet for maternal PKU syndrome in PKU mice, although resulting in perinatal survival, also results in most pups dying in the postnatal weaning period (21). Our results also suggest that differences in reproductive success depend on the age at which hyperphenyalaninemia is controlled. Both dopamine and serotonin, the neurotransmitter products of Tyr and Trp, play important roles in brain development, maternal behavior, and neonate behavior. All of these factors may affect maternal PKU syndrome in mice. Our results show that pegvaliase therapy resulted in a successful, if incomplete, rescue of maternal PKU syndrome in PKU mice. Pegvaliase treatment of human maternal PKU syndrome is likely not subject to the physiological factors that limited treatment in the PKU mouse. Given clinical data from our facility, and others, which show the need for additional options, we believe that if safety standards for use of pegvaliase in human pregnancy can be established, reduction of blood Phe levels by enzyme substitution therapy may become an additional treatment for maternal PKU syndrome.
SUPPLEMENTARY MATERIAL

Supplementary material is linked to the online version of the paper at http://www.nature.com/pr

\section{ACKNOWLEDGMENTS}

We acknowledge Carlos Santamaria and Bin Zhao for their dedicated analysis of plasma pharmacokinetic samples, and Sean Bell, Paul Fitzpatrick, and Kim Fellows-Peake for providing the pegvaliase for these experiments.

\section{STATEMENT OF FINANCIAL SUPPORT}

The retrospective clinical review of patient records was supported by the Department of Pediatrics, UF College of Medicine. Dr Laipis's laboratory has been the recipient of grants from BioMarin Pharmaceutical, which supported the PKU mouse studies described in this manuscript. DGM, CAO'N, and TLST are employees of BioMarin Pharmaceutical Inc.

Disclosure: Dr Laipis has no financial ties to BioMarin Pharmaceutical other than research funding. Dr Zori and McCune are consultants for BioMarin Pharmaceutical, and directed the Phase II and Phase III trials of rAvPAL-PEG in human patients carried out at the UF College of Medicine, also supported by BioMarin Pharmaceutical. They have no further conflicts of interest.

\section{REFERENCES}

1. Blau N, van Spronsen FJ, Levy HL. Phenylketonuria. Lancet 2010;376: 1417-27.

2. Waisbren SE, Noel K, Fahrbach K, et al. Phenylalanine blood levels and clinical outcomes in phenylketonuria: a systematic literature review and meta-analysis. Mol Genet Metab 2007;92:63-70.

3. Bickel H, Gerrard J, Hickmans EM. The influence of phenylalanine intake on the chemistry and behaviour of a phenyl-ketonuric child. Acta Paediatr 1954;43:64-77.

4. Paul D. A double-edged sword. Nature 2000;405:515.

5. Koch R, Hanley W, Levy $\mathrm{H}$, et al. A preliminary report of the collaborative study of maternal phenylketonuria in the United States and Canada. J Inherit Metab Dis 1990;13:641-50.

6. Rouse B, Azen C, Koch R, et al. Maternal Phenylketonuria Collaborative Study (MPKUCS) offspring: facial anomalies, malformations, and early neurological sequelae. Am J Med Genet 1997;69:89-95.

7. Waisbren SE, Chang P, Levy HL, et al. Neonatal neurological assessment of offspring in maternal phenylketonuria. J Inherit Metab Dis 1998;21: $39-48$.

8. Waisbren SE, Azen C. Cognitive and behavioral development in maternal phenylketonuria offspring. Pediatrics 2003;112:1544-7.

9. Widaman KF, Azen C. Relation of prenatal phenylalanine exposure to infant and childhood cognitive outcomes: results from the International Maternal PKU Collaborative Study. Pediatrics 2003;112:1537-43.

10. Rouse B, Matalon R, Koch R, et al. Maternal phenylketonuria syndrome: congenital heart defects, microcephaly, and developmental outcomes. J Pediatr 2000;136:57-61.

11. Enns GM, Koch R, Brumm V, Blakely E, Suter R, Jurecki E. Suboptimal outcomes in patients with PKU treated early with diet alone: revisiting the evidence. Mol Genet Metab 2010;101:99-109.

12. Koch R, Trefz F, Waisbren S. Psychosocial issues and outcomes in maternal PKU. Mol Genet Metab 2010;99 (Suppl 1): S68-74.

13. Lenke RR, Levy HL. Maternal phenylketonuria and hyperphenylalaninemia. An international survey of the outcome of untreated and treated pregnancies. N Engl J Med 1980;303:1202-8.

14. Koch R, Hanley W, Levy H, et al. The Maternal Phenylketonuria International Study (1984-2002). Pediatrics 2003;112:1523-9.

15. Koch R, Hanley W, Levy $H$, et al. Maternal phenylketonuria: an international study. Mol Genet Metab 2000;71:233-9.

16. Matalon R, Michals K, Gleason L. Maternal PKU: strategies for dietary treatment and monitoring compliance. Ann NY Acad Sci 1986;477: $223-30$. 


\section{Articles | zeile et al.}

17. Lee PJ, Ridout D, Walter JH, Cockburn F. Maternal phenylketonuria: Report from the United Kingdom Registry 1978-97. Arch Dis Child 2005;90:143-6.

18. Maillot F, Lilburn M, Baudin J, Morley DW, Lee PJ. Factors influencing outcomes in the offspring of mothers with phenylketonuria during pregnancy: the importance of variation in maternal blood phenylalanine. Am J Clin Nutr 2008;88:700-5.

19. Fisch RO. Comments on diet and compliance in phenylketonuria. Eur J Pediatr 2000;159 (Suppl 2): S142-4.

20. Wang L, Gamez A, Archer H, et al. Structural and biochemical characterization of the therapeutic Anabaena variabilis phenylalanine ammonia lyase. J Mol Biol 2008;380:623-35.

21. Shedlovsky A, McDonald JD, Symula D, Dove WF. Mouse models of human phenylketonuria. Genetics 1993;134:1205-10.

22. Martynyuk AE, Ucar DA, Yang DD, et al. Epilepsy in phenylketonuria: a complex dependence on serum phenylalanine levels. Epilepsia 2007;48: 1143-50.

23. Sarkissian CN, Gámez A, Wang L, et al. Preclinical evaluation of multiple species of PEGylated recombinant phenylalanine ammonia lyase for the treatment of phenylketonuria. Proc Natl Acad Sci USA 2008;105: 20894-9.

24. McCaman MW, Robins E. Fluorimetric method for the determination of phenylalanine in serum. J Lab Clin Med 1962;59:885-90.

25. Schulpis KH, Tjamouranis J, Karikas GA, Michelakakis H, Tsakiris S. In vivo effects of high phenylalanine blood levels on $\mathrm{Na}^{+}, \mathrm{K}^{+}$-ATPase, $\mathrm{Mg}^{2+}$-ATPase activities and biogenic amine concentrations in phenylketonuria. Clin Biochem 2002;35:281-5.

26. Longo N, Harding CO, Burton BK, et al. Single-dose, subcutaneous recombinant phenylalanine ammonia lyase conjugated with polyethylene glycol in adult patients with phenylketonuria: an open-label, multicentre, phase 1 dose-escalation trial. Lancet 2014;384: 37-44.
27. Shiman R, Gray DW. Formation and fate of tyrosine. Intracellular partitioning of newly synthesized tyrosine in mammalian liver. J Biol Chem 1998;273:34760-9.

28. Zhou Q-Y, Palmiter RD. Dopamine-deficient mice are severely hypoactive, adipsic, and aphagic. Cell 1995;83:1197-209.

29. Yang S, Lee YJ, Kim JM, et al. A murine model for human sepiapterinreductase deficiency. Am J Hum Genet. 2006;78:575-87.

30. Miller SM, Lonstein JS. Dopaminergic projections to the medial preoptic area of postpartum rats. Neuroscience 2009;159:1384-96.

31. Pascucci T, Andolina D, Ventura R, Puglisi-Allegra S, Cabib S. Reduced availability of brain amines during critical phases of postnatal development in a genetic mouse model of cognitive delay. Brain Res 2008;1217:232-8.

32. Andolina D, Conversi D, Cabib S, et al. 5-Hydroxytryptophan during critical postnatal period improves cognitive performances and promotes dendritic spine maturation in genetic mouse model of phenylketonuria. Int J Neuropsychopharmacol 2010;1:1-11.

(c) (1) () $\odot$ This work is licensed under a Creative Commons Attribution-NonCommercial-NoDerivs 4.0 International License. The images or other third party material in this article are included in the article's Creative Commons license, unless indicated otherwise in the credit line; if the material is not included under the Creative Commons license, users will need to obtain permission from the license holder to reproduce the material. To view a copy of this license, visit http://creativecommons.org/licenses/ by-nc-nd/4.0/

C) The Author(s) (2018) 